15

\title{
Комплекс SOLPEX для исследования излучения Солнца в мягком рентгеновском диапазоне волн
}

\author{
(C) C.В. Кузин, ${ }^{1}$ A.C. Кириченко, ${ }^{1}$ M. Stęślicki, ${ }^{2}$ J. Sylwester, ${ }^{2}$ M. Siarkowski, ${ }^{2}$ Ż. Szaforz, ${ }^{2}$ S. Płocieniak, ${ }^{2}$ \\ J. Bąkała, ${ }^{2}$ J. Barylak, ${ }^{2}$ P. Podgórski, ${ }^{2}$ D. Ścisłowski, ${ }^{2}$ M. Kowaliński, ${ }^{2}$ C.A. Богачев, ${ }^{1}$ A.A. Перцов ${ }^{1}$ \\ ${ }^{1}$ Физический институт им. П.Н. Лебедева РАН, \\ 119991 Москва, Россия \\ ${ }^{2}$ Отделение фризики Солнца института космических исследований ПАН, \\ Вроцлав, Польша \\ e-mail: s.kuzin@lebedev.ru
}

Поступило в Редакцию 28 марта 2019 г.

В окончательной редакции 28 марта 2019г.

Принято к публикации 15 апреля 2019г.

B комплекс SOLPEX входят 2 инструмента, для регистрации мягкого рентгеновского излучения Солнца, входящих в аппаратуру „КОРТЕС“, которая будет установлена на борту Международной космической станции. Первый - быстровращающийся многокристальный брэгговский спектрометр, предназначенный для регистрации солнечных спектров в диапазоне $0.4-23 \AA$ с временным разрешением не хуже $0.1 \mathrm{~s}$. Второй инструмент представляет собой камеру-обскуру с фокусным расстоянием $58 \mathrm{~cm}$. Инструмент имеет поле зрения $2 \times 2 \mathrm{deg}$ и угловое разрешение $2 \mathrm{arg}$. min, временное разрешение до $0.2 \mathrm{~s}$. Энергетический диапазон определяется входным фильтром и составляет $1-10 \mathrm{KeV}$, а энергетическое разрешение $-0.5 \mathrm{KeV}$. Комбинация этих двух инструментов позволяет локализовать горячие солнечные источники в короне, определять их скорость и проводить спектральную диагностику.

Ключевые слова: рентгеновская спектроскопия, солнечная корона, брэгговский спектрометр, камераобскура.

DOI: 10.21883/JTF.2019.12.48478.123-19

\section{Введение}

Исследования Солнца в мягком рентгеновском диапазоне спектра начались практически одновременно с началом эры космических исследований. Первый эксперимент по регистрации коротковолнового излучения солнечной короны был проведен в 1957 г. на втором искусственном спутнике Земли [1]. В 1963-1965 гг. были получены первые рентгеновские изображения Солнца в сериях советских и американских экспериментов с использование камер-обскур [2,3]. По мере прогресса в физике Солнца, направленность космических солнечных исследований в 70-80 гг. прошлого века сместилась в спектроскопию. Это обусловлено тем, что в большинстве активных процессов в солнечной короне температура плазмы достигает нескольких миллионов градусов, в результате чего подавляющая часть энергии электромагнитного диапазона выделяется в мягкой рентгеновской (MP) области в линиях многозарядных ионов с высокой степенью ионизации. С этого времени МР спектроскопия плазмы стала мощным инструментом для исследования свойств процессов энерговыделения в солнечной короне. Была проведена серия космических экспериментов, в которой были получены значимые результаты по солнечной физике [4-6]. В настоящее время с развитием оптики вакуумного ультрафиолетового $($ ВУФ) и МР диапазонов, интерес к МР спектроскопии снизился, но она до сих пор остается значимым инструментом исследования процессов в корональной плазме.

\section{Комплекс аппаратуры „Кортес“}

В ФИАН разрабатывается комплекс аппаратуры „Кортес“, предназначенный для изучения солнечной короны на международной космической станции [7]. „Кортес“ является многоканальным инструментом и включает в себя 8 независимо работающих приборов (табл. 1).

Начало работы на орбите аппаратуры „Кортес“ в настоящее время планируется на 2024 г., что соответствует максимуму солнечной активности. Поэтому основными задачами эксперимента является исследование вспышечных процессов на Солнце, которые характеризуются большими объемами выделяемой энергии, быстрыми временами развития и высокими температурами плазмы. Исследования планируется проводить методом изображающей спектроскопии, который заключается в регистрации этих процессов одновременно спектральными и изображающими приборами. Так как вспышки на Солнце довольно трудно предсказуемы, для их регистрации будет реализован квазинепрерывный режим наблюдения полного диска Солнца с высоким временным разрешением.

Телескопы Т1-T3 предназначены для регистрации структур в широком диапазоне температур, от 20 тыс. градусов до $1.4 \mathrm{млн.} \mathrm{градусов,} \mathrm{и} \mathrm{соответствующих} \mathrm{им}$ высот от хромосферы до ближней короны. Особенностью телескопа Т1 является то, что в этом спектральном диапазоне находится интенсивная линия иона Fe XXIV 
Таблица 1. Состав комплекса аппаратуры „Кортес“

\begin{tabular}{|c|c|c|}
\hline Канал & $\begin{array}{c}\text { Спектральный } \\
\text { диапазон }\end{array}$ & Назначение \\
\hline Телескоп Т1 & $195 \AA$ & $\begin{array}{l}\text { Изображения короны } \\
\text { и вспышек }\end{array}$ \\
\hline Телескоп Т2 & $304 \AA$ & $\begin{array}{l}\text { Изображения } \\
\text { переходного слоя }\end{array}$ \\
\hline Телескоп Т3 & $584 \AA$ & $\begin{array}{l}\text { Изображения } \\
\text { хромосферы }\end{array}$ \\
\hline $\begin{array}{l}\text { Спектрогелиограф } \\
\text { C1 }\end{array}$ & $180-210 \AA$ & $\begin{array}{l}\text { Спектры полного } \\
\text { диска Солнца }\end{array}$ \\
\hline $\begin{array}{l}\text { Спектрогелиограф } \\
\text { С2 }\end{array}$ & $280-335 \AA$ & $\begin{array}{l}\text { Спектры полного } \\
\text { диска Солнца }\end{array}$ \\
\hline $\begin{array}{l}\text { Спектрогелиограф } \\
\mathrm{Mg}\end{array}$ & $8.42 \AA$ & $\begin{array}{l}\text { Изображения вспышек } \\
\text { и активных областей } \\
\text { в линии иона MgXII }\end{array}$ \\
\hline Спектрометр RDS & $0.3-22.8 \AA$ & $\begin{array}{l}\text { Спектры вспышек } \\
\text { и активных областей }\end{array}$ \\
\hline Камера-обскура РНІ & $0.5-15 \mathrm{KeV}$ & $\begin{array}{l}\text { Изображения вспышек } \\
\text { и активных областей }\end{array}$ \\
\hline
\end{tabular}

с температурой возбуждения 10 млн. градусов, характерной для вспышек. Таким образом, этот канал является необходимым для комплексного исследования вспышек с помощью спектрогелиографов и спектрометров.

Спектрогелиографы $\mathrm{C} 1$ и $\mathrm{C} 2$ регистрируют полный диск Солнца. Они имеют спектральное разрешение около $0.03 \AA$ на ячейку и пространственное - около $3 \mathrm{arg}$. s в перпендикулярном направлении. Благодаря наблюдению всего диска Солнца регистрация спектров вспышек с помощью таких инструментов гораздо более информативна и удобна, чем с помощью щелевых спектрометров. При этом информация о пространственной конфигурации исследуемых структур будет получена из телескопов Т1 и Т2.

Спектрогелиограф $\mathrm{Mg}$ является монохроматическим прибором, регистрирующим изображение солнечных структур с температурой выше 4 млн. градусов в линии дублета MgXII. Значимость этого канала заключается в том, что он локализует положение только горячих источников в короне.

Спектрометр RDS и камера-обскура PHI объединены в комплекс SOLPEX, который разрабатывается в отделении физики Солнца польской академии наук [8]. Весь комплекс „Кортес“ управляется бортовым компьютером, размещенным внутри герметичного отсека служебного модуля российского сегмента МКС.

\section{Вращающийся барабанный спектрометр RDS}

Вращающийся барабанный спектрометр RDS предназначен для получения спектров Солнца в диапазоне

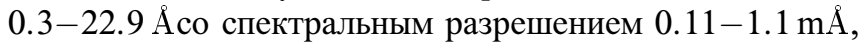
в зависимости от поддиапазона, и временным разреше- нием около $0.1 \mathrm{~s}$. Регистрируемый спектральный диапазон включает в основном линии многозарядных ионов с высокой степенью ионизации, которая характерна для вспышечных процессов. Спектральная информация RDS будет дополняться спектральной информацией спектрогелиографов С1 и С2, изображениями телескопа Т1, спектрогелиографа $\mathrm{Mg}$ и камеры-обскуры PHI.

Принцип действия инструмента основан на брэгговской дифракции рентгеновского излучения на кристаллах. В RDS 4 пары плоских кристаллов установлены на вращающийся барабан. Дифрагированное излучение регистрируется с помощью кремниевых твердотельных детекторов SDD. Электронный тракт прибора позволяет регистрировать точное положение пары кристаллов и время регистрации каждого фотона, что дает информацию о его энергии.

Схема прибора представлена на рис. 1. Типы используемых в спектрометре кристаллов и соответствующие им спектральные диапазоны представлены в табл. 2. В качестве детекторов будут использованы SDD (silicon drift detector): 1 шт. (передний детектор) KETEK VITUS H18LE с окном из полимера AP3.3 и 3 шт. KETEK VITUS H50 с окном из бериллия толщиной $12.5 \mu \mathrm{m}$.

\section{Камера-обскура PHI}

Камера-обскура является простейшим оптическим инструментом, входная апертура которого представляет собой небольшое отверстие в непрозрачном материале. Камера-обскура имеет ряд преимуществ перед другими оптическими системами: простота изготовления и юстировки, надежность, отсутствие хроматических аберраций и независящее от спектра пропускание.

Хотя камеры-обскуры для рентгеновской астрономии применялись еще в 60-х годах прошлого века $[2,3]$, их применение в современных исследованиях может быть востребовано в случае использования детектора с

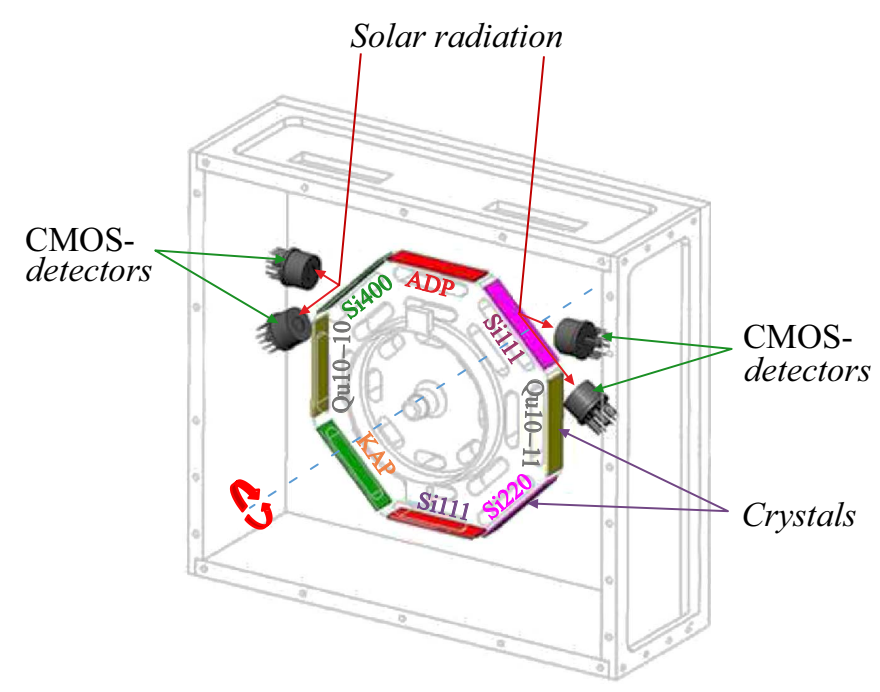

Рис. 1. Схема прибора RDS. 
Таблица 2. Параметры каналов спектрометра RDS

\begin{tabular}{|c|c|c|c|c|c|}
\hline $\begin{array}{c}\text { Канал } \\
\text { (количество кристаллов) }\end{array}$ & $\begin{array}{l}\text { Спектральный } \\
\text { диапазон }(\AA)\end{array}$ & 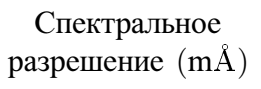 & Кристалл & Срез & $2 \mathrm{~d}(\AA)$ \\
\hline \multicolumn{6}{|c|}{ Передний детектор } \\
\hline $\begin{array}{l}1(1) \\
2(1) \\
3(2) \\
4(1) \\
5(1) \\
6(1) \\
7(1)\end{array}$ & $\begin{array}{c}1.678-2.330 \\
2.375-3.295 \\
3.878-5.381 \\
4.133-5.735 \\
5.265-7.306 \\
6.585-9.137 \\
16.474-22.859\end{array}$ & $\begin{array}{c}0.11 \\
0.15 \\
0.25 \\
0.27 \\
1.3 \\
1.3 \\
6.5\end{array}$ & $\begin{array}{c}\mathrm{Si} \\
\mathrm{Si} \\
\mathrm{Si} \\
\text { Кварц } \\
\text { Кварц } \\
\mathrm{ADP} \\
\mathrm{KAP}\end{array}$ & $\begin{array}{c}400 \\
220 \\
111 \\
10-11 \\
10-10 \\
101 \\
001\end{array}$ & \begin{tabular}{c|}
2.715 \\
3.840 \\
6.2715 \\
6.684 \\
8.514 \\
10.648 \\
26.640
\end{tabular} \\
\hline \multicolumn{6}{|c|}{ Задний детектор } \\
\hline $\begin{array}{l}1(1) \\
2(1) \\
3(2) \\
4(1) \\
5(1) \\
6(1) \\
7(1)\end{array}$ & $\begin{array}{c}0.442-1.618 \\
0.625-2.288 \\
1.021-3.737 \\
1.088-3.983 \\
1.386-5.073 \\
1.734-6.345 \\
4.337-15.875\end{array}$ & $\begin{array}{c}0.15 \\
0.22 \\
0.36 \\
0.38 \\
0.49 \\
0.61 \\
1.5\end{array}$ & $\begin{array}{c}\mathrm{Si} \\
\mathrm{Si} \\
\mathrm{Si} \\
\text { Кварц } \\
\text { Кварц } \\
\mathrm{ADP} \\
\mathrm{KAP}\end{array}$ & $\begin{array}{c}400 \\
220 \\
111 \\
10-11 \\
10-10 \\
101 \\
001\end{array}$ & $\begin{array}{c}2.715 \\
3.840 \\
6.2715 \\
6.684 \\
8.514 \\
10.648 \\
26.640\end{array}$ \\
\hline
\end{tabular}

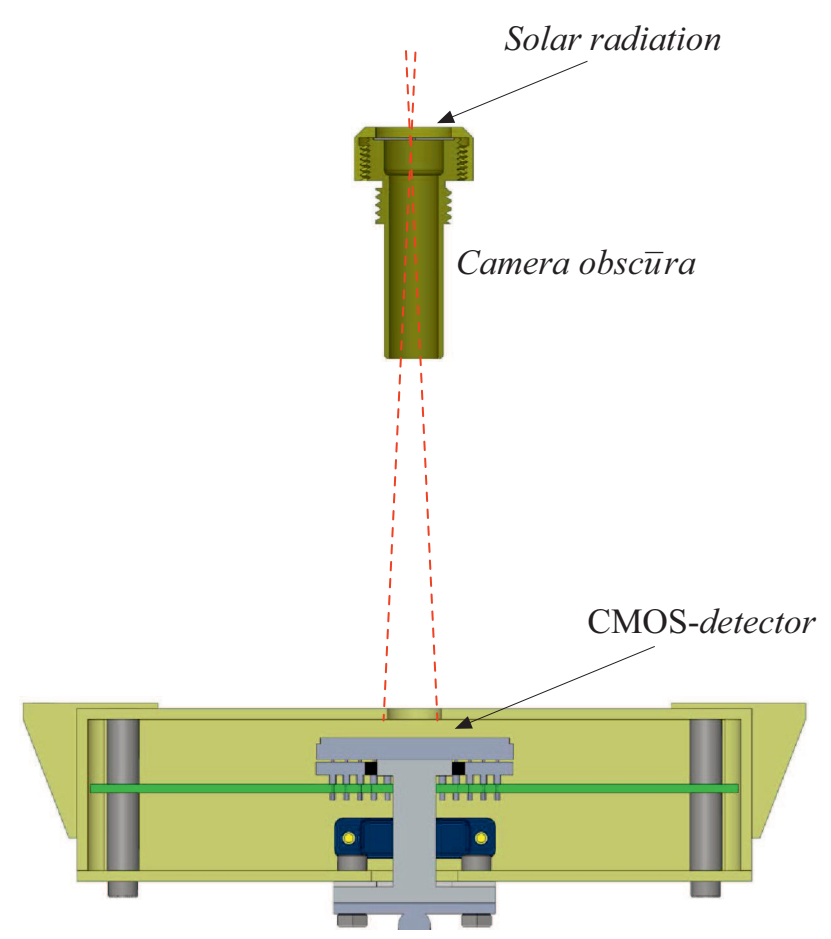

Рис. 2. Схема прибора PHI.

энергетическим разрешением и калиброванной эффективностью регистрации. Таким образом, камера-обскура позволяет одновременно получать изображения Солнца, спектр излучения, формирующего изображение, и его фотометрию.

Именно такой подход реализован в канале РНІ, схема которого представлена на рис. 2. Ключевым элементом является детектор, регистрирующий рентгеновское излучение. В качестве детектора выбран CMOS-сенсор компании Gpixel Gsense 400BSI, форматом $2048 \times 2048$ ячеек, размер ячейки $11 \times 11 \mu \mathrm{m}$. Измерения показали, что этот детектор имеет энергетическое разрешение около $0.5 \mathrm{KeV}$ в диапазоне $1-10 \mathrm{KeV}$. Особенность данного сенсора является его короткое время считывания (коло $0.2 \mathrm{~s}$ ) и высокая радиационная стойкость, что позволяет отказаться от механического затвора при регистрации изображений.

Входная апертура размещается на расстоянии $580 \mathrm{~mm}$ от плоскости детектора и представляет собой свинцовый диск с отверстием диаметра $0.7 \mathrm{~mm}$, закрытым полиамидной пленкой толщиной $15 \mu \mathrm{m}$. Такая конфигурация PHI обеспечивает поле зрения $2 \times 2^{\circ}$ и угловое разрешение около $20 \mathrm{arg}$. s.

Основным назначением прибора является локализация горячих источников, их фотометрия и получение дополнительной спектральной информации.

\section{Заключение}

Приборы RDS и PHI, входящие в комплекс SOLPEX инструмента „Кортес“, являются развитием традиционной техники рентгеновской изображающей спектроскопии. Комбинация этих инструментов позволяет получать изображения активных процессов на Солнце одновременно со спектральной информацией о плазме, формируемой в результате этих процессов. Такое сочетание крайне важно для анализа трансформации энергии во время этих процессов, определения их мощности, уточнения химического состава плазмы. Высокое временное разрешение этих инструментов позволит исследовать динамику вспышек и ассоциированных со вспышками процессов в солнечной короне. 


\section{Финансирование работы}

Работа выполнена при поддержке Российского научного фонда (грант 17-12-01567) и Poland National Science Centre UMO 0 2917/25/B/ST9/01821.

\section{Конфликт интересов}

Авторы заявляют, что у них нет конфликта интересов.

\section{Список литературы}

[1] Мандельштам С.Л., Тиндо И.П., Воронько Ю.К. // Искусственные спутники Земли. 1961. Вып. 11.

[2] Житник И.А., Крутов В.В., Малявкин Л.П. и др. // Космические исследования. Т. 2. 1964. № 6. С. 920.

[3] Blake R.L., Chubb T.A., Friedman H., Unzicker A.E. // Astrophys. J. 1963. Vol. 137. P. 3.

[4] Neupert W.M., Swartz M. // Bulletin of the American Astronomical Society. 1970. Vol. 2. P. 211.

[5] Grineva Yu.I., Karev V.I., Korneev V.V., Krutov V.V. et al. // Solar Physics. 1973. Vol. 29. N 2. P. 441.

[6] Lang K.R., Willson R.F., Smith K.L. et al. // Astrophys. J. 1987. Vol. 322. N 15. P. 1035.

[7] Vishnyakov E.A., Bogachev S.A., Kirichenko A.S. et al. // Proceedings of the SPIE, EUV and X-Ray Optics: Synergy Between Laboratory and Space. 2017. Vol. 10235.

[8] Sylwester J., Stesślicki M., Bakała J. et al. // Exp Astron. 2019. DOI: 10.1007/s10686-018-09618-4https://doi.org/10.1007/s10686-018-09618-4 